\title{
Shear-enhanced yield stress in electrorheological fluids
}

\author{
Kai Chi Lau, Lihong Shi, Wing Yim Tam, and Ping Sheng \\ Department of Physics, The Hong Kong University of Science and Technology, Clear Water Bay, Kowloon, Hong Kong
}

(Received 10 December 2002; published 14 May 2003)

\begin{abstract}
We report the enhancement of yield stress in electrorheological (ER) fluids by a shear-annealing method, using creep-recovery $(\mathrm{CR})$ cycles under an external electric field. The enhancement depends on the duration and the strength of the applied shear stress, as well as on the number of the CR cycles. The shear-annealing method enables the particles in the ER fluid to form microstructures with an increased yield stress, manifest as better aligned and denser columns. For a sufficiently large number of CR cycles, with an optimal combination of stress duration and shear strength, a stable state can be obtained whereby shear deformation becomes elastic.
\end{abstract}

DOI: 10.1103/PhysRevE.67.052502

PACS number(s): 62.10.+s, 47.17.+e, 83.60.La

Electrorheological (ER) fluid is a complex fluid consisting of micron-sized dielectric particles dispersed in a nonconducting fluid. Its rheological property can be controlled by the application of an external electric field whereby the particles first form, in milliseconds, chains and then columns along the electric field direction [1]. These chains and columns enable the ER fluid to resist shear deformation. The stronger the electric field, the stronger the resistance to shear. More importantly the process is reversible by the removal of the electric field, thus making the ER fluid an ideal material for real time feedback control applications in isolation dampers, clutches, and valves [1]. However, in order to meet the demanding requirements for practical applications, the strength of the ER fluid must be improved. The strength of an ER fluid depends on two factors: the material and the microstructures inside the columns. In the past, almost all efforts had focused on the materials part [2-5]. However, it is known that the microstructure and size of the columns can play an important role, in particular, for the static case. It is now known that the ideal microstructure for monodispersed spherical-particle ER fluid, as well as its counterpart, the magnetorheological (MR) fluid, is a body-centeredtetragonal (BCT) lattice $[4,6,7]$. However, in real experiments the formation of BCT columns is far from perfect because of random particle aggregation upon the application of an electric field. Recently, it has been reported that by compressing the MR fluid under an external magnetic field, the strength of the MR fluid can be increased dramatically [8]. Here, we show the strength of ER fluid to be similarly enhanced through shear-annealing, using creep-recovery (CR) cycles [9]. It is possible to produce an elastic end state after the application of a sufficient number of CR cycles.

We used $2.5 \pm 0.1 \mu \mathrm{m}$ silica spheres dispersed in silicon oil as the ER fluid [10]. The silica particles were used without removing the water residue, and the particle volume fraction was 0.45 . A Hakke CS-20 rheometer with an ER option and parallel plate sensor was used. The ER fluid was first mixed using a shaker for half an hour to ensure uniform mixing. It was then transferred to the rheometer with temperature of the parallel plate senor regulated at $25^{\circ} \mathrm{C}$ by using a thermal circulator. A $700-\mathrm{Hz}$ ac electric field, up to $1.5 \mathrm{kV} / \mathrm{mm}$, was applied between the plates with a $2-3 \mathrm{~mm}$ spacing. The ER sample was "conditioned" at a constant strain rate for $1 \mathrm{~min}$ before applying the electric field. CR cycles were then applied to the sample after applying the electric field. One CR cycle consisted of a constant shear stress period and a recovery period without shear stress, with the applied electric field on. The strength of the shear stress,
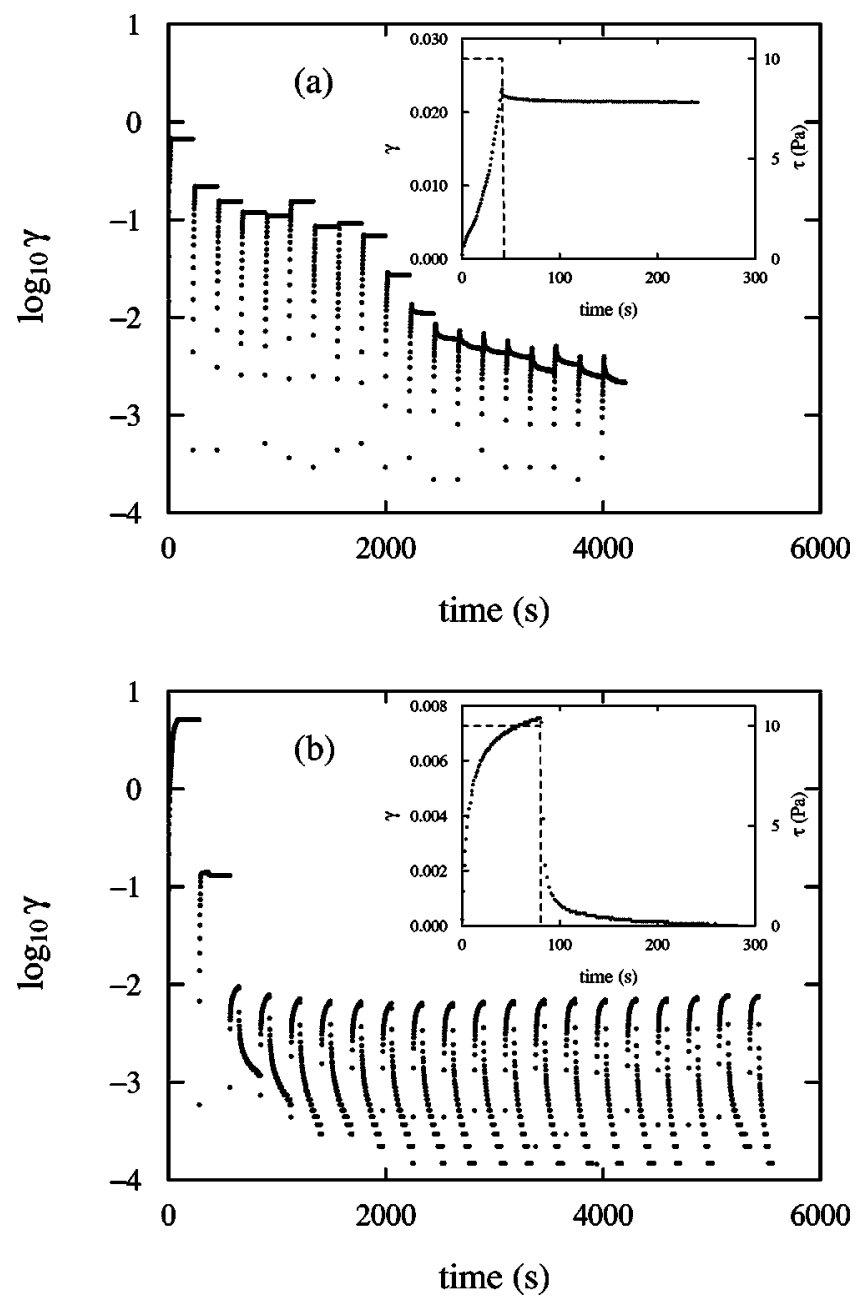

FIG. 1. The log of the strain $\gamma$ (deformation) of ER fluid containing 0.45 volume fraction of $2.5 \mu \mathrm{m}$ silica spheres in silicon oil under a $700-\mathrm{Hz} 1.0-\mathrm{kV} / \mathrm{mm}$ electric field for $20 \mathrm{CR}$ cycles using a $10 \mathrm{~Pa}$ shear stress and a shearing period of (a) $40 \mathrm{~s}$ and (b) $80 \mathrm{~s}$. The insets show the deformation (solid dots) and the shear stress $\tau$ (dashed line) of one CR cycle. 

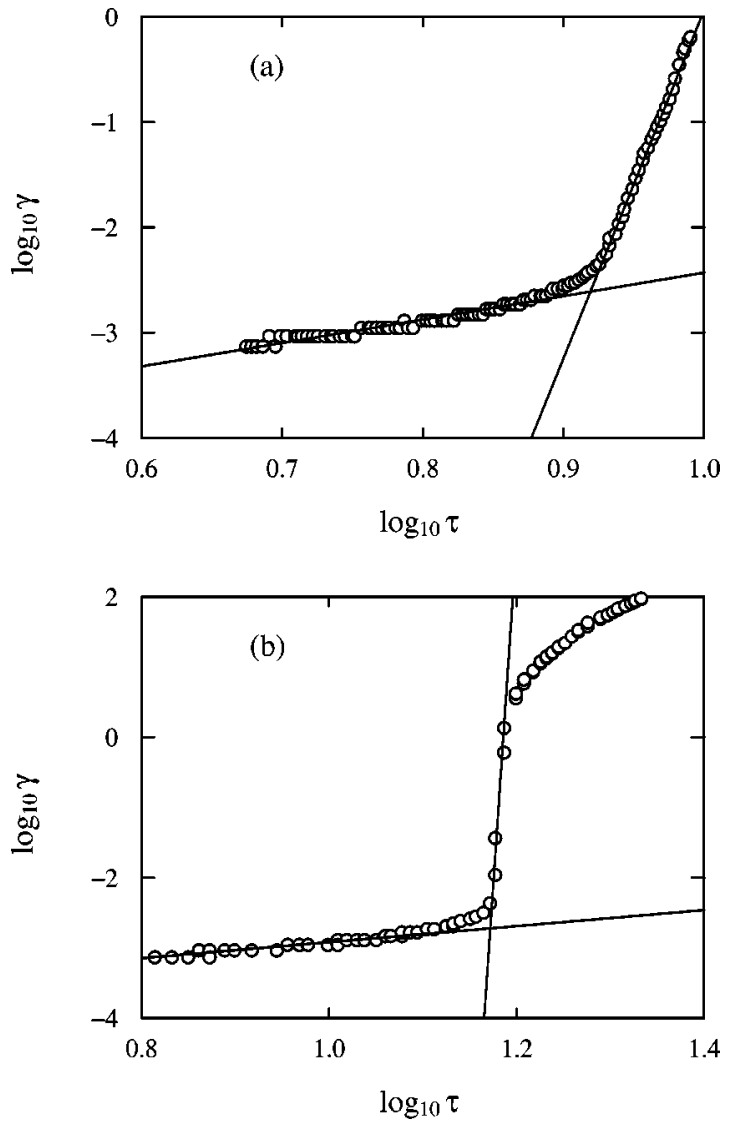

FIG. 2. Strain $\gamma$ (deformation) versus stress $\tau$ in $\log$ scale for (a) random initial condition and (b) few CR cycles as shown in Fig. 1. The solid lines in (a) are tangents of the two segments. The intercept of these two lines gives the yield stress of the ER fluid.

the shearing period, and the recovery period were varied to study their effects. Figure 1 shows the strain $\gamma$ (deformation in $\log$ scale) in response to $\mathrm{CR}$ cycles using a $1-\mathrm{kV} / \mathrm{mm}$ electric field and a shear stress $\tau$ of $10 \mathrm{~Pa}$, which was slightly larger than the yield stress of the ER fluid with a random initial condition without the CR cycles. Note that the deformation is offset to zero after a complete CR cycle. In Fig. 1(a) the stress and recovery periods are $40 \mathrm{~s}$ and $200 \mathrm{~s}$, respectively. The inset shows the deformation (solid dots) and stress (dashed line) of a CR cycle. In Fig. 1(a), it is clear that the deformation is mostly permanent, with a small elastic portion. The overall deformation amplitude decreases after repeated $\mathrm{CR}$ cycles, but there does not seem to be a steady state after $20 \mathrm{CR}$ cycles. In contrast, for longer shearing periods, the behavior is very different as shown in Fig. 1(b) for $80 \mathrm{~s}$ shearing period. It is seen that after a few CR cycles an elastic steady state was reached where deformation returns to the origin after the removal of the stress [see the inset to Fig. 1(b)]. Elastic steady state could also be obtained for a longer shearing periods up to $160 \mathrm{~s}$. For shearing periods longer than $160 \mathrm{~s}$, the ER fluid started to flow and no state steady can be obtained.

It is clear that the CR cycles affect the structure of the ER fluid. When the ER fluid with random initial condition is put under an electric field, chains and columns are formed. However, their internal structures are far from perfect. Under the
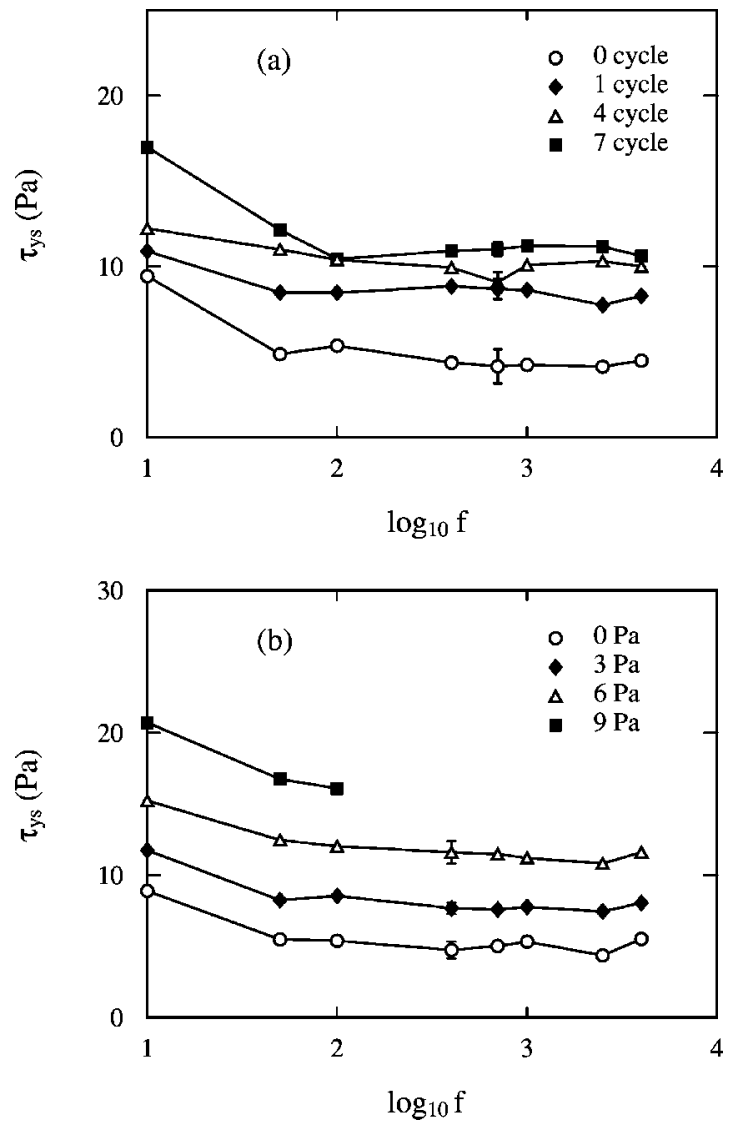

FIG. 3. The frequency dependence of yield stress $\tau_{y s}$ of ER fluid after CR cycles under a $400-\mathrm{Hz} 1.5-\mathrm{kV} / \mathrm{mm}$ electric field with $160 \mathrm{~s}$ shearing period and $200 \mathrm{~s}$ recovery period for (a) various numbers of CR cycles at fixed shear stress of $5 \mathrm{~Pa}$ (0 cycle corresponds to yield stress measured with random initial conditions) and (b) different shear stress with $5 \mathrm{CR}$ cycles. $0 \mathrm{~Pa}$ corresponds to no shear stress in the CR cycles.

shear stress of a CR cycle, chains and columns were stretched and tilted. This process is envisioned to remove some of the defects by slippings and restructurings. After the stress is removed (in the recovery period), the chains and columns would relax and combine to form larger columns. Hence a CR cycle is similar to an annealing process. By repeating the $\mathrm{CR}$ cycles, the structure of the ER fluid could become stronger, resulting in a larger ER effect.

To measure the yield stress of the ER fluid, the deformation resulting from a slow (control) ramping in stress was measured. A log-log plot of deformation versus stress will have two segments as shown in Fig. 2(a) for random initial conditions. The first segment represents the elastic deformation below the yield point, while the second segment corresponds to flow above the yield point. The yield stress can be defined as the intersection of the two segments as indicated in Figs. 2(a) and 2(b).

Figure 3(a) shows the shear yield stress $\tau_{y s}$ as a function of frequency (of the applied electric field) for various numbers of $\mathrm{CR}$ cycles (using a shear of $5 \mathrm{~Pa}$ in the CR cycles with an electric field of $1.5 \mathrm{kV} / \mathrm{mm}$ at $400 \mathrm{~Hz}$ ). The enhancement due to $\mathrm{CR}$ cycles is obvious: more $\mathrm{CR}$ cycles imply larger enhancement. A maximum enhancement factor of 5 


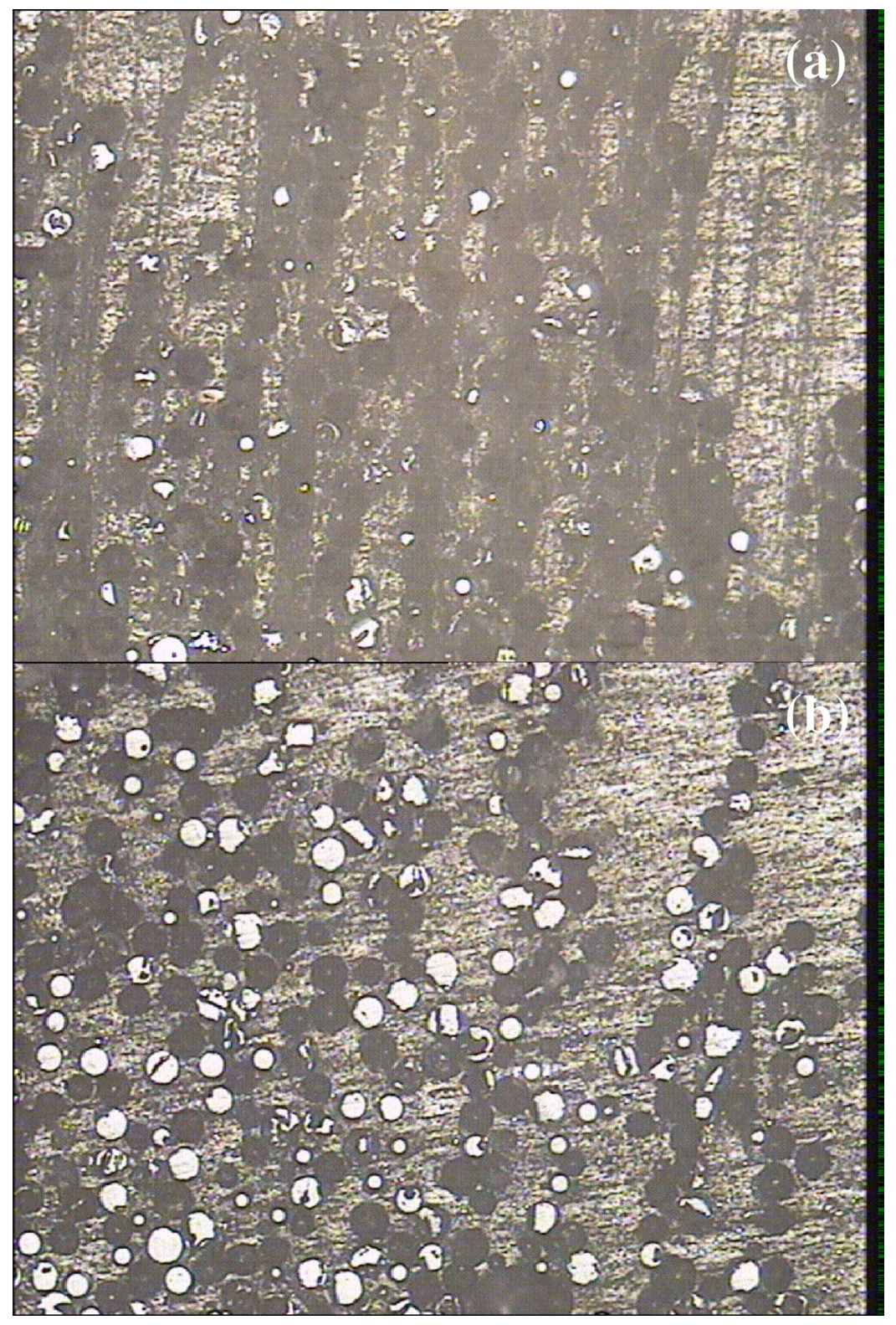

FIG. 4. Cross section along the $E$ field direction for $50 \mu \mathrm{m}$ glass spheres in epoxy ER fluid with particle volume fraction 0.46 and $1-\mathrm{kV} / \mathrm{mm}$ $E$ field for (a) with and (b) without the CR cycles. $50 \mu \mathrm{m}$ spheres were used because of easy observation as compared to $2.5 \mu \mathrm{m}$ particles. The epoxy based system was more complicated than the oil based system because of the hardening effect of the epoxy during the CR cycles. The viscosity of the epoxy changed from about $100 \mathrm{cps}$ to much higher values at the later CR cycles as the epoxy hardened, and thus hindered the effectiveness of the CR cycles. Nevertheless, the epoxy based ER fluid showed similar stress-strain behavior as the oil based ER fluids in the early CR cycles as in Fig. 1(b), resulting in better chain/ column formations than without the CR cycles. was obtained [not shown in Fig. 3(a)] using $40 \mathrm{CR}$ cycles at $400 \mathrm{~Hz}$ electric field. However, further increase in CR cycles did not lead to further improvement. Figure 3(b) shows the shear yield stress as a function of shear stress used in the CR cycles. It is seen that the larger the shear stress used in the $\mathrm{CR}$ cycles, the larger the enhancement. Furthermore, the dependence is approximately linear. For stress of $9 \mathrm{~Pa}$, the sample flowed during the CR cycles and hence no yield stress measurement was attempted at high frequencies. For even larger stress, the sample flowed for all frequencies.

The above results can be explained by the reformation of the ER fluids microstructure under the CR cycles. Under a constant shear in a CR cycle, chains or columns were stretched and tilted, leading to particle slipping or permanent deformation. However, once stress is removed in the recovery period, chains and columns would recombine to form larger and more regular columns. By repeating the $C R$ cycles, the structure of the ER fluid will tend to be more regular and stable as shown in Fig. 4 the cross section of the columns along the $E$ field direction for an ER fluid frozen in epoxy with [Fig. 4(a)] and without [Fig. 4(b)] the CR cycles. It is obvious that Fig. 4(a) with CR cycles shows much denser and well-packed columns than Fig. 4(b) without the $\mathrm{CR}$ cycles. For a sufficient number of CR cycles, it is possible for the ER fluid to reach a state where deformation becomes elastic. The optimal strength of stress, shearing, and recovery periods, and the number of CR cycles would depend very much on the properties of different ER systems. However, it is clear that the shear stress used cannot be much larger than the yield stress of the ER fluid with random initial conditions. Also the stress period should not be too long, or otherwise the chains and columns will have no chance to reform. To conclude, we have shown that using a shearannealing method, the strength of ER fluid can be enhanced. The enhancement is due to structure reformation.

This work was supported by the Competitive Earmarked Research Grant No. HKUST 6122/98P of the Research Grant Council of Hong Kong. 
[1] K.O. Havelka and F.E. Filisko, Progress in Electrorheology (Plenum Press, New York, 1995); W.M. Winslow, J. Appl. Phys. 20, 1137 (1949).

[2] A. Inoue, in Electrorheology Fluids, edited by J.D. Carlson, A.F. Sprecher, and H. Conrad (Technomic, Lancaser, 1989), p. 176.

[3] W.Y. Tam, G. Yi, W. Wen, H. Ma, M.M.T. Loy, and P. Sheng, Phys. Rev. Lett. 78, 2987 (1997).

[4] W. Wen, N. Wang, H. Ma, Z. Lin, W.Y. Tam, C.T. Chan, and P. Sheng, Phys. Rev. Lett. 82, 4248 (1999).

[5] W. Wen, Y. Zhang, Z. Huang, W. Ge, and P. Sheng (private communications).

[6] R. Tao and J.M. Sun, Phys. Rev. Lett. 67, 398 (1991); R. Tao and J.M. Sun, Phys. Rev. A 44, R6181 (1991).
[7] G. Bossis, H. Clercx, Y. Grasselli, and E. Lemaice, in Electrorheological Fluids, edited by R. Tao and G.D. Roy (World Scientific, Singapore, 1994), p. 153; H.J.H. Clercx and G. Bossis, Phys. Rev. E 48, 2721 (1993); L.C. Davis, Phys. Rev. A 46, R719 (1992); H. Ma, W. Wen, W.Y. Tam, and P. Sheng, Phys. Rev. Lett. 77, 2499 (1996).

[8] X. Tang, X. Zhang, and R. Tao, J. Appl. Phys. 87, 2634 (2000).

[9] The effect of Creep-recovery cycle on ER fluids has been reported recently, see, e.g., Y. Otsubo and K. Edamura, J. Colloid Interface Sci. 172, 530 (1995); Colloids Surf., A 109, 63 (1996).

[10] The $2.5 \mu \mathrm{m}$ silca sphere was obtained from Nippon Shokubai and the silicon oil was sample No. 705 from DOW Corning. 